\title{
BMJ Open Recruitment feasibility to a cohort study of endocrine and metabolic health among survivors of childhood brain tumours: a report from the Canadian study of Determinants of Endometabolic Health in ChIlDrEn (CanDECIDE)
}

M Constantine Samaan, ${ }^{1,2}$ Katrin Scheinemann, ${ }^{1,3,4}$ Sarah Burrow, ${ }^{5}$ Rejane F Dillenburg, ${ }^{1,6}$ Ronald D Barr, ${ }^{1,3}$ Kuan-Wen Wang, ${ }^{1,2}$ Marlie Valencia, ${ }^{1,2}$ Lehana Thabane ${ }^{1,7,8,9,10}$

To cite: Samaan MC Scheinemann K, Burrow S, et al. Recruitment feasibility to a cohort study of endocrine and metabolic health among survivors of childhood brain tumours: a report from the Canadian study of Determinants of Endometabolic Health in ChIIDrEn (CanDECIDE). BMJ Open 2014;4:e05295. doi:10.1136/bmjopen-2014005295

- Prepublication history for this paper is available online. To view these files please visit the journal online (http://dx.doi.org/10.1136/ bmjopen-2014-005295).

Received 19 March 2014 Revised 5 June 2014 Accepted 10 June 2014

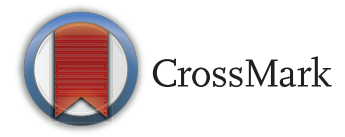

For numbered affiliations see end of article.

Correspondence to Dr M Constantine Samaan; samaanc@mcmaster.ca

\section{ABSTRACT}

Objectives: The aim of this study was to test the feasibility of recruitment and performance of study procedures of the Canadian Study of Determinants of Endometabolic Health in ChIIDrEn (CanDECIDE) study, which was designed to assess the determinants of endocrine and metabolic health in survivors of childhood brain tumours.

Setting: A single paediatric tertiary care centre in Hamilton, Ontario, Canada.

Participants: We included boys and girls, aged 5 years and older, who were lean (body mass index (BMI) below 85th centile for age and gender) or overweight/obese (BMI 85th centile or above for age and gender). We excluded children on steroids or immunosuppressant therapy, smokers and those who had an active infection for the 2 weeks prior to participation.

Outcomes: Feasibility targets included recruitment rate of at least $50 \%$, the consenting of $80 \%$ of participants to provide biological samples, $90 \%$ questionnaire completion rate and the ability to process biological samples from at least $80 \%$ of participants.

Results: We approached 210 potential participants, and of the $112(53 \%)$ who agreed to participate, 30 $(26.8 \%)$ completed the study visit over 7 months. All participants agreed to fast, provide biological samples and complete the questionnaires. Sample collection was successful in 97\% (29/30) of participants and laboratory procedures were feasible in $100 \%$ of collected samples. We also tested resources required for the conduct of the full study including personnel, space, laboratory equipment and procedures and determined that they are all feasible.

Conclusions: Recruitment and consenting of patients for the CanDECIDE study may be feasible. However, we are considering prolonging recruitment duration and
Strengths and limitations of this study

- We tested the feasibility of the Canadian Study of Determinants of Endometabolic Health in ChIIDrEn study that is examining the endometabolic health of survivors of childhood brain tumours.

- We established feasibility of study procedures including consenting, questionnaire administration, sample collection and laboratory procedures.

- We approached 210 potential participants, and of the $112(53 \%)$ who agreed to participate, 30 $(26.8 \%)$ completed the study visit over 7 months. which is lower than the predicted rate of $50 \%$.

- We are proceeding with the full study with prolongation of the duration of recruitment and consideration for inclusion of other centres to meet study targets.

collaboration with other centres to meet recruitment targets due to lower than expected recruitment rate. Completion of questionnaires and implementation of sample processing protocols are feasible.

\section{INTRODUCTION}

Advances in brain tumour diagnosis and therapy have resulted in high survival rates in children over the past three decades, yet this has been coupled with higher premature mortality, and considerable morbidity, including obesity and diabetes, in survivors. ${ }^{1-6}$ The factors that contribute to the metabolic problems in survivors of childhood brain tumours (SCBT) include hypothalamic damage, ${ }^{7-9}$ 
deficiency of pituitary hormones, ${ }^{7} 810$ reduced mobility and physical activity levels, ${ }^{11-14}$ reduced exercise capacity and motivation, ${ }^{15}{ }^{16}$ sleep disturbances,${ }^{17}$ visual compromise, imbalance and pain ${ }^{18} 19$ and medication use (eg, steroids, antidepressants). ${ }^{20}$ Defining the mechanisms of obesity and diabetes in survivors will help design treatment and prevention strategies that will lead to improved lifespan and quality of life.

Over the past few years, new insights into the role of the immune system in obesity and cancer have been described. Tumour cells interact with immune cells to create the tumour microenvironment that regulates tumour cell growth, metastasis and metabolism. ${ }^{21}{ }^{22}$ One of the immune cells involved in this interaction is the monocyte, which migrates to tumours and neighbouring areas and differentiates to tumour-associated macrophages. ${ }^{21}$ While these cells have been detected in brain tumours, their role in systemic inflammation, obesity and diabetes in survivors is unknown. ${ }^{2324}$

Obesity is characterised by both local and systemic inflammatory responses. ${ }^{25}$ 26 Macrophages are present within metabolic organs and secrete cytokines that propagate local inflammation, ${ }^{27-31}$ and these cytokines can enter the circulation causing systemic inflammation. ${ }^{32-35}$ In turn, inflammation causes insulin resistance and, if the latter is untreated, will lead to type 2 diabetes. ${ }^{31}$

There is very limited evidence available regarding systemic inflammation in SCBT. In a study including a small group of brain tumour survivors $(n=19)$, SCBT had elevation of the inflammatory marker $\mathrm{C}$ reactive protein (CRP) ${ }^{36}$ but CRP is not a satisfactory marker of inflammation in children. ${ }^{37}{ }^{38}$ In the general paediatric population with no history of cancer, our group ${ }^{39}$ and others have reported some of the cytokines associated with childhood obesity. ${ }^{40-45}$

In order to establish if obese SCBT have enhanced systemic inflammation compared to lean survivors and non-cancer controls, and to identify the potential lifestyle factors that may drive this response, the Canadian Study of Determinants of Endometabolic Health in ChIlDrEn (CanDECIDE study) was initiated. The protocol for the study has already been published, ${ }^{46}$ and the aim of this pilot study was to evaluate study processes and procedures to determine feasibility of the full study.

The primary aim of this pilot study was to test the feasibility of the CanDECIDE study including recruitment rates, data collection forms' content and design, data completion, time needed to interview participants, feasibility of fasting and biological sample collection. In addition, we wanted to test laboratory procedures to process samples including time, space, equipment, personnel, consumables and additional resources needed.

\section{METHODS}

Setting and participants

This study was initiated at McMaster Children's Hospital, a tertiary paediatric centre in Hamilton, Ontario,
Canada. SCBT were recruited from the neurooncology clinics at the Hospital, and description of the setting has been published previously. ${ }^{46}$ The control group included children with healed fractures, minor scoliosis or those with a history of syncope with no structural cardiac or conduction defects (recruited from clinics at the Hospital), along with healthy children recruited from the local community.

\section{Inclusion criteria}

We included boys and girls, aged 5 years and older, who were lean (BMI below 85th centile for age and gender) or overweight/obese (BMI 85th centile or above for age and gender) and for SCBT we included those who had completed tumour therapy at least 6 months earlier.

\section{Exclusion criteria}

We excluded children on steroids or immunosuppressant therapy, smokers and those who had an active infection for the 2 weeks prior to participation. ${ }^{46}$

\section{Data collection and instruments}

We used standardised tools to collect data comprising diet, ${ }^{47}{ }^{48}$ physical activity, ${ }^{49}$ sleep,${ }^{50}$ stress ${ }^{51}$ and neighbourhood walkability. ${ }^{52}$

Additional data collected include age, sex, ethnicity, education, profession, social history, medical history, medications, illicit drug use, alcohol consumption, smoking, birth history, menstrual history in girls and family history. We designed a Case Report Form specifically for the study, and collected data including diagnosis, location of tumour, treatment(s), comorbidities, height, weight, waist circumference, hip circumference, BMI, blood pressure, heart rate and grip strength. ${ }^{46}$ Adiposity was measured using bioelectrical impedance with Tanita scale (Tanita Corporation $)^{53}$ for those less than 18 years of age, and the InBody520 body composition analyser (GE Healthcare) for those 18 years or older. ${ }^{54}$

\section{Sample collection and laboratory processing}

All blood and urine samples were collected in the fasting state. Fasting was started from midnight for at least $8 \mathrm{~h}$. Owing to the age group the study is dealing with, children were allowed to drink water but sugary drinks and food were not allowed until sample collection was completed. Experienced paediatric phlebotomists and/or researchers collected blood samples, and samples were processed within two hours of collection. For saliva, participants either gave fasting samples, or had breakfast and then waited for at least 30 minutes before providing the samples using Oragene saliva collection kits (DNA genotek, Ontario, Canada) as recommended by the manufacturer.

\section{Feasibility of study procedures}

The primary outcome for this study was to evaluate the feasibility of the full CanDECIDE study. Specifically, we examined the following: 
1. The feasibility of recruitment by calculating the number of participants approached and rates of acceptance and consent;

2. Case report form and questionnaire completion rates;

3. The evaluation of resources needed to conduct the study including personnel, space and laboratory equipment;

4. The feasibility of fasting and biological sample collection: The latter was noted by agreement to provide blood, urine and saliva samples;

5. The feasibility of analytes' isolation and processing in the laboratory including monocytes, serum, plasma, buffy coat, urine and saliva.

\section{Feasibility criteria}

In this feasibility study, we designed a priori criteria for a feasible study which included the following conditions: recruitment rate of at least $50 \%$, the consenting of $80 \%$ of participants to provide biological samples, $90 \%$ completion rate of all questionnaires and the ability to isolate monocytes, serum, plasma, buffy coat, urine and saliva from at least $80 \%$ of samples. We also evaluated the resources needed to perform the study, including personnel, space and laboratory utilisation for sample processing.

\section{Statistical analysis}

The primary reason for this pilot study was to establish feasibility. The target sample size was $\mathrm{n}=30$ which was determined primarily based on feasibility considerations to test procedures for the full study where we plan to recruit 300 participants. The sociodemographic and outcome variables are reported using descriptive measures including the mean (SD) for continuous variables and number (percent) for categorical variables. The study data were managed using Research Electronic Data Capture (REDCap) tools hosted at McMaster University. ${ }^{55}$ The analysis of feasibility outcomes was descriptive in nature, with the results expressed as percentages and feasibility assessed against the corresponding targets. The data were analysed using SPSS V.22.0 (IBM Corp, Armonk, New York, USA).

\section{RESULTS}

\section{Participant recruitment rate}

Over the period from November 2012-July 2013, 210 potential participants agreed to be approached by the research team after their care provider asked for permission; in the case of participants from the community, they contacted the study team directly having heard about the study. Of those participants, 112 (53\%) agreed to participate including 1 from the community, 23 from neuro-oncology, 11 from cardiology and 77 from orthopaedic clinics. There were no major differences in recruitment rates between neuro-oncology and other clinics. Participants who did not wish to participate in the study $(\mathrm{n}=98)$ cited different reasons for their decision including travel distance and transportation $(n=26)$, discomfort with blood sampling $(n=24)$, no interest in research participation $(n=32)$, unavailability to attend research clinic visits due to scheduling conflicts $(n=13)$ and other reasons $(n=3)$. For this pilot study, we completed the recruitment of 30 participants within the above period, resulting in a recruitment rate of $26.8 \%$ (figure 1).

\section{Participant demographics}

As shown in table 1 , of the 30 participants recruited 12 $(n=4$ female) were in the SCBT group and $18(n=12$ female) in the control group. The majority of participants in both groups $(83 \%)$ were Caucasian Europeans. In the SCBT group, the average age was $14.3 \pm 4.6$ years, and $33 \%$ of SCBT were overweight/obese. One-third had received radiotherapy, $31 \%$ chemotherapy, $83 \%$ had surgery and the majority received short-term steroids in the perioperative period. The tumour types included five astrocytomas, five medulloblastomas and two other types. The mean (SD) age in the non-cancer comparison group was 12.6 (2.7) years, of whom $39 \%$ were overweight/obese.

The availability of clinical space was paramount to allow study visits to take place. We did utilise clinic space consisting of five clinic rooms within the hospital space, and we used four rooms to interview participants and one room was set up for blood collection.

All those who agreed to participate and signed consent forms agreed to provide biological samples. We did offer all participants the use of topical anaesthetic patches for phlebotomy, and only a few opted to use them. Once collected, the samples were then taken to the research laboratory for further processing. In one patient, it was not possible to get blood samples, giving a sample collection rate of $97 \%$. The equipment utilisation in the laboratory was appropriate for the workload of this study, and four research students were trained in the laboratory study procedures and were responsible for sample processing, aliquoting, storage and cataloguing.

\section{Biological sample isolation and processing in the laboratory}

The following samples were processed:

Monocytes: We isolated monocytes from whole blood collected into a $15 \mathrm{~mL}$ Corning tube containing $150 \mu \mathrm{L}$ of $100 \mathrm{mM}$ EDTA. The samples were layered on polymorphprep separation gradient (Cedarlane Labs, Oakville, Canada), and samples centrifuged at $650 \mathrm{~g}$ for $35 \mathrm{~min}$ at room temperature with brakes off. To isolate monocytes, we removed the mononuclear cell layer from the gradient and used EasySep magnetic bead Human Monocyte Enrichment Kit without CD16 depletion (Stemcell Technologies, Vancouver, Canada) as per manufacturer's instructions. 
Figure 1 Flow diagram for CanDECIDE study recruitment.

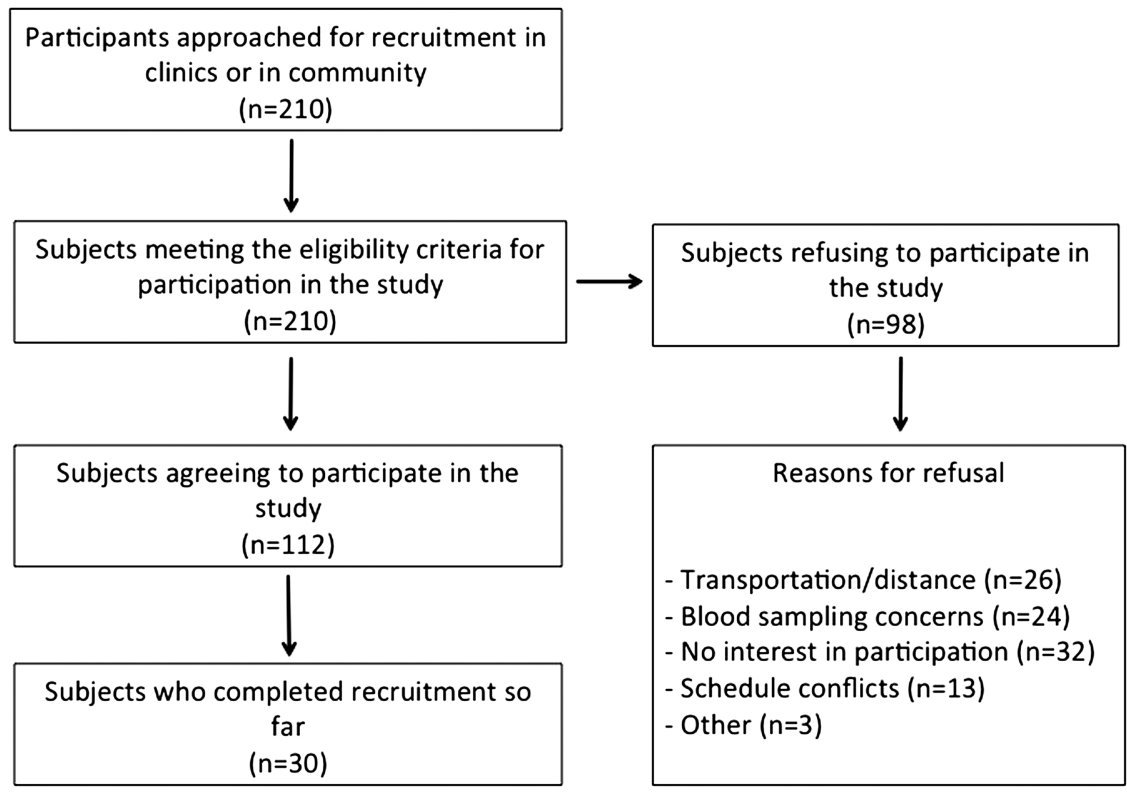

Serum: Fasting serum samples were collected from clotted blood after centrifugation for $15 \mathrm{~min}$ at $1500 \mathrm{~g}$ at room temperature. Samples were aliquoted and stored at $-80^{\circ} \mathrm{C}$.

Plasma: Fasting plasma samples were collected by centrifuging EDTA-treated blood at $1500 \mathrm{~g}$ for $15 \mathrm{~min}$ at room temperature. Samples were aliquoted and stored at $-80^{\circ} \mathrm{C}$ until further use.

Buffy coat: EDTA-treated blood samples were centrifuged at $1500 \mathrm{~g}$ for $15 \mathrm{~min}$ at room temperature, and the buffy coat layer was collected. Samples were stored at $-80^{\circ} \mathrm{C}$ until further use.

Urine: Fasting urine samples were collected in $90 \mathrm{~mL}$ urine containers, then aliquoted and frozen at $-80^{\circ} \mathrm{C}$.

\begin{tabular}{|c|c|c|c|c|}
\hline \multirow[b]{2}{*}{ Variable } & \multicolumn{2}{|c|}{$\begin{array}{l}\text { Control } \\
(n=18)\end{array}$} & \multicolumn{2}{|c|}{$\begin{array}{l}\text { SCBT } \\
(n=12)\end{array}$} \\
\hline & Mean & SD & Mean & SD \\
\hline Age (years) & 12.6 & 2.7 & 14.3 & 4.6 \\
\hline Height $(\mathrm{cm})$ & 156.7 & 11.6 & 151.5 & 23.6 \\
\hline Weight (kg) & 54.0 & 15.0 & 52.5 & 24.0 \\
\hline $\mathrm{BMI}\left(\mathrm{kg} / \mathrm{m}^{2}\right)$ & 21.7 & 4.6 & 21.8 & 6.7 \\
\hline Per cent overweight/obesity & 39.0 & & 33.0 & \\
\hline Per cent fat mass & 24.1 & 8.6 & 23.3 & 13.0 \\
\hline Waist circumference $(\mathrm{cm})$ & 67.3 & 16.4 & 70.4 & 22.1 \\
\hline Hip circumference $(\mathrm{cm})$ & 83.5 & 16.3 & 82.1 & 23.2 \\
\hline Heart rate (bpm) & 78.9 & 9.5 & 85.5 & 10.6 \\
\hline Systolic BP (mm Hg) & 107.9 & 9.5 & 99.3 & 11.6 \\
\hline Diastolic BP (mm Hg) & 65.1 & 8.1 & 64.2 & 7.8 \\
\hline Birth weight (g) & 3550 & 598 & 3611 & 657 \\
\hline
\end{tabular}

$\mathrm{BMI}$, body mass index; BP, blood pressure; Control, participants with no history of cancer; SCBT, survivors of childhood brain tumours.
Saliva: Saliva samples were stored at room temperature until further use.

\section{Feasibility results}

In this study, we approached potential participants over a 7-month period. The number of those who agreed to participate was $112(53 \%)$. So far, we have completed the study on 30 participants giving a recruitment rate of $26.8 \%$, which is lower than our target of $50 \%$.

Case report form completion was carried out in $100 \%$ of cases; we used a system by which the first researcher who interviews the participant would handover the questionnaires to another researcher who checks all forms and completes any missing data while participants are in clinic. In addition, the forms were audited within 1 week of study completion, and families and participants contacted to clarify or add any missing information. The completion of all study procedures including consenting, completion of questionnaires and provision of biological samples took 70-90 min.

In relation to clinic staffing, we had eight research students involved in the recruitment of participants, and this number was judged adequate to deal with the workload, based on the need to cover clinics during the week for recruitment and staffing of the research clinic visits. These students were provided with specific training to approach potential participants, obtaining consent, scheduling of clinic visits, conducting study procedures including anthropometric and other measures and the administration of study questionnaires. In addition, three students from the group who were not involved in study recruitment or procedures were responsible for auditing the questionnaires and completion of data collection forms to ensure high quality of data. Furthermore, two other students were involved in data entry into REDCap database. 
The techniques used to isolate the study analytes proved to be feasible. Monocyte isolation yielded excellent results in $90 \%$ of cases; in three participants, the gradient centrifugation did not separate the cells accurately so no sample could be retrieved. Serum samples from two participants showed evidence of haemolysis and were labelled as such until further processing is undertaken to determine if this has interfered with sample quality. One saliva sample had food particles, and in another one the preservative fluid leaked after sample collection, hence these samples will be excluded from further analysis.

\section{DISCUSSION}

This feasibility study tested the clinical and laboratory procedures for the CanDECIDE study, a cohort study designed to determine the factors affecting the endocrine and metabolic outcomes in SCBT and comparing them to participants with no history of cancer. Using the initial 30 participants' data, we demonstrate the relatively lower rates of recruitment than anticipated initially, adequate responses to questionnaires and satisfactory consent rates to provide biological samples. We also demonstrate the feasibility of additional study procedures including consenting, data collection, entry and analysis. We also demonstrate the feasibility of sample collection and laboratory sample processing protocols and techniques.

\section{Study strengths}

One of the significant strengths of this study was the establishment of collaborations among several clinical teams within the Hospital to recruit study participants. The alliance created by this collaboration demonstrates the feasibility of the expansion of this platform to conduct the full study. As we serve 2.3 million people in our catchment area, this represents close to $8 \%$ of the whole population of Canada, which is a representative population of children.

In addition, the creation of robust data collection tools, body measurement protocols and standardised platforms for data handling has allowed the study group to collect high quality data and demonstrated the feasibility of this structure for use in the full study with no modifications.

\section{Lessons learned to inform the design of CanDECIDE study}

One of the limitations we faced during this pilot phase was related to recruitment targets. We envisaged initially recruiting 300 participants over 2 years; this was based on $50 \%$ projected recruitment rate, and seeing around three patients per week. The recruitment rate for the feasibility study was $26.8 \%$, as scheduling these patients to clinic visits was taking longer than we had anticipated at first. This was because sometimes the team could not use the phone number or email address provided to contact the family, or scheduling conflicts for families prevented them from attending study visits and they needed to reschedule. To address this, our next step is to prolong the recruitment phase to 4 years to try to address this issue. In addition, we had to broaden the inclusion of other types of brain tumours, as we were focusing only on astrocytomas, medulloblastomas and ependymomas. The caveat to this is increased heterogeneity of the samples, which may then require a larger sample size to achieve sufficient power to detect meaningful differences in inflammatory marker levels. One possible avenue that may shorten the recruitment duration and maintain homogeneity of the sample is collaboration with other centres caring for SCBT. We have already started discussions with other neurooncology centres within Canada and abroad, and this approach may help circumvent the issue of recruitment rates. As we forge collaborations with other neurooncology centres, our sample will certainly be more representative of paediatric populations in industrialised countries.

Ultimately, the challenge is to get the neurooncology patients to participate in the study, and then to match the comparison group to those who participate. At this point, the groups are different, and this is a reflection of the early stage of the study. We will ensure that the groups are more homogeneous moving forward by periodically balancing the two groups through ensuring that the comparison group is age-matched and sex-matched to the survivors group.

Another approach that we will adopt is to broaden community access by advertising in schools and recreational facilities. We will also add incentives including paying for costs of parking and providing small tokens for participation.

The one question that remains unanswered is the retention rate of participants in the full study that will run for 10 years, especially the non-brain tumour group. For the SCBT group, we have a unique setting in our hospital whereby children and adults are followed in the same clinic, which allows us to maintain relatively high rates of longitudinal follow-up.

In order to enhance retention in the study, we are planning to have newsletters sent to participants annually via email and mail; we are also planning annual study information days in which participants and their families are invited to engage the research team, receive study updates and address any questions about various aspects of the study.

The study may also be prone to several sources of bias. Selection bias may occur if the choice of participants is not representative of the general population, as our comparison group is mostly composed of children attending other clinics in the Hospital. Our approach to minimise selection bias is to ensure that recruited participants are free from disease or have a self-limiting condition. For example, participants recruited from the orthopaedic clinic are seen in research study visits after their fractures are healed; our recruits from the cardiology clinic were assessed for cardiac defects and only 
included if they had a structurally normal heart and no conduction defects. Recruits from the community were again assessed to determine the absence of disease and medication use that may affect their immune-metabolic status before consenting.

Recall bias may arise when questions are asked about events that occurred a long time ago, for example, family history details of grandparents. For SCBT, all clinical data regarding care have been obtained from clinical notes from a previous study we conducted thus minimising this form of bias.

Another form of bias we need to guard against is social desirability bias, which arises when participants offer 'healthier' answers to the researcher. One of the ways we circumvented this was by using structured data collection tools and laboratory data (eg, looking at levels of fasting glucose or fasting lipid levels) to ensure we can account for this issue.

\section{CONCLUSIONS}

In summary, we have tested the feasibility of the CanDECIDE study, and found that many study procedures are accomplishable. However, we will need to prolong the recruitment phase, broaden tumour types included in the study, and consider incorporating other centres into the study. In addition, we validated our laboratory procedures for sample processing and we are currently preparing to proceed with the full study with implementation of the changes suggested.

\author{
Author affiliations \\ ${ }^{1}$ Department of Pediatrics, McMaster University, Hamilton, Ontario, Canada \\ 2Division of Pediatric Endocrinology, McMaster Children's Hospital, Hamilton, \\ Ontario, Canada \\ ${ }^{3}$ Division of Pediatric Hematology/Oncology, McMaster Children's Hospital, \\ Hamilton, Ontario, Canada \\ ${ }^{4}$ Department of Paediatric Hematology/Oncology, University Children's \\ Hospital, Münster, Germany \\ ${ }^{5}$ Division of Pediatric Orthopedics, Department of Pediatric Surgery, McMaster \\ University, Hamilton, Ontario, Canada \\ ${ }^{6}$ Division of Pediatric Cardiology, McMaster Children's Hospital, Hamilton, \\ Ontario, Canada \\ ${ }^{7}$ Department of Clinical Epidemiology \& Biostatistics, McMaster University, \\ Hamilton, Ontario, Canada \\ ${ }^{8}$ Department of Anesthesia, McMaster University, Hamilton, Ontario, Canada \\ ${ }^{9}$ Centre for Evaluation of Medicines, Hamilton, Ontario, Canada \\ ${ }^{10}$ Biostatistics Unit, St Joseph's Healthcare-Hamilton, Hamilton, Ontario, \\ Canada
}

Acknowledgements The authors would like to acknowledge the study participants and their families for contributing to the study. The authors also acknowledge the McMaster University students who helped with study visit conduct and laboratory procedures: Ms Amrita Amarnaney, Ms Pauline Chang, Ms Connie Cheung, Ms Jiyeh Joo, Mr Ishan Aditya, Mr Nicola Sahar, Mr Ameir Makar, Mr Calvin Tang, Ms Glara Rhee, Ms Brittany Watson and Ms Madeleine Bondy.

Contributors MCS conceived the study question and generated the hypotheses. MCS, LT, RDB, RFD, SB and KS finalised the study design. MCS, KWW, MV and LT completed the statistical analysis plans. MCS, RDB, SB, RFD and KS contributed to the definition of study cohorts, inclusion and exclusion criteria, recruitment plan and study logistics including space and resource allocation. KWW and MV were involved in recruitment, consenting, administration of questionnaires, anthropometric measures, data collation and analysis. MCS wrote the manuscript and all authors reviewed the current version.

Funding This study was partly funded by Joshua's Journey (Hamilton Health Sciences Foundation) Neurooncology fund.

Competing interests MCS is funded by New Investigator Fund grant from Hamilton Health Sciences.

Ethics approval Hamilton Integrated Health Research Board.

Provenance and peer review Not commissioned; externally peer reviewed.

Data sharing statement The additional data include additional clinical data based on the questionnaires reported in our protocol paper including diet, physical activity, sleep, stress and build environment. This paper is focused on reporting feasibility, and the data will be included in the full study for analyses and publications. As the data collection is ongoing, and data are not fully analysed, the data will be available at the end of the study to collaborators in an agreed on format at that point in time.

Open Access This is an Open Access article distributed in accordance with the Creative Commons Attribution Non Commercial (CC BY-NC 4.0) license, which permits others to distribute, remix, adapt, build upon this work noncommercially, and license their derivative works on different terms, provided the original work is properly cited and the use is non-commercial. See: http:// creativecommons.org/licenses/by-nc/4.0/

\section{REFERENCES}

1. Nader PR, O'Brien M, Houts $R$, et al. Identifying risk for obesity in early childhood. Pediatrics 2006;118:e594-601.

2. Freedman DS, Mei Z, Srinivasan SR, et al. Cardiovascular risk factors and excess adiposity among overweight children and adolescents: the Bogalusa Heart Study. J Pediatr 2007;150:12-17. e2.

3. Lustig RH, Post SR, Srivannaboon K, et al. Risk factors for the development of obesity in children surviving brain tumors. J Clin Endocrinol Metab 2003;88:611-16.

4. Pui CH, Pei D, Pappo AS, et al. Treatment outcomes in black and white children with cancer: results from the SEER database and St Jude Children's Research Hospital, 1992 through 2007. J Clin Oncol 2012;30:2005-12.

5. The Canadian Cancer Registry. Statistics Canada. Custom tabulation (1992-2007) F. 2011.

6. Kaatsch P. Epidemiology of childhood cancer. Cancer Treatment Rev 2010;36:277-85.

7. Cohen LE. Endocrine late effects of cancer treatment. Curr Opin Pediatr 2003;15:3-9.

8. Cohen LE. Endocrine late effects of cancer treatment. Endocrinol Metab Clin North Am 2005;34:769.

9. Lustig $\mathrm{RH}$, Hinds PS, Ringwald-Smith K, et al. Octreotide therapy of pediatric hypothalamic obesity: a double-blind, placebo-controlled trial. J Clin Endocrinol Metab 2003;88:2586-92.

10. Oberfield SE, Sklar CA. Endocrine sequelae in survivors of childhood cancer. Adolesc Med 2002;13:161--9, viii.

11. Schmitz KH, Holtzman J, Courneya KS, et al. Controlled physical activity trials in cancer survivors: a systematic review and meta-analysis. Cancer Epidemiol Biomarkers Prev 2005;14:1588-95.

12. Miller TL, Lipsitz SR, Lopez-Mitnik G et al. Characteristics and determinants of adiposity in pediatric cancer survivors. Cancer Epidemiol Biomarkers Prev 2010;19:2013-22.

13. Armstrong GT, Conklin HM, Huang S, et al. Survival and long-term health and cognitive outcomes after low-grade glioma. Neuro Oncol 2011;13:223-34.

14. Zeltzer LK, Recklitis C, Buchbinder D, et al. Psychological status in childhood cancer survivors: a report from the Childhood Cancer Survivor Study. J Clin Oncol 2009;27:2396-404.

15. Mertens AC, Yasui $\mathrm{Y}$, Liu Y, et al. Pulmonary complications in survivors of childhood and adolescent cancer. A report from the Childhood Cancer Survivor Study. Cancer 2002;95:2431-41.

16. Mulrooney DA, Yeazel MW, Kawashima T, et al. Cardiac outcomes in a cohort of adult survivors of childhood and adolescent cancer: retrospective analysis of the Childhood Cancer Survivor Study cohort. BMJ 2009;339:b4606.

17. Mulrooney DA, Ness KK, Neglia JP, et al. Fatigue and sleep disturbance in adult survivors of childhood cancer: a report from the childhood cancer survivor study (CCSS). Sleep 2008;31:271. 
18. Geenen MM, Cardous-Ubbink MC, Kremer LC, et al. Medical assessment of adverse health outcomes in long-term survivors of childhood cancer. JAMA 2007;297:2705-15.

19. Pietilä S, Mäkipernaa A, Sievänen $\mathrm{H}$, et al. Obesity and metabolic changes are common in young childhood brain tumor survivors. Pediatr Blood Cancer 2009;52:853-9.

20. Green DM, Cox CL, Zhu L, et al. Risk factors for obesity in adult survivors of childhood cancer: a report from the Childhood Cancer Survivor Study. J Clin Oncol 2012;30:246-55.

21. Solinas G, Germano G, Mantovani A, et al. Tumor-associated macrophages (TAM) as major players of the cancer-related inflammation. J Leukocyte Biol 2009;86:1065-73.

22. Coussens LM, Werb Z. Inflammation and cancer. Nature 2002;420:860-7.

23. Kushchayev SV, Sankar T, Eggink LL, et al. Monocyte galactose/ $\mathrm{N}$-acetylgalactosamine-specific C-type lectin receptor stimulant immunotherapy of an experimental glioma. Part II: combination with external radiation improves survival. Cancer Manag Res 2012;4:325-34

24. Kushchayev SV, Sankar T, Eggink LL, et al. Monocyte galactose/ $\mathrm{N}$-acetylgalactosamine-specific C-type lectin receptor stimulant immunotherapy of an experimental glioma. Part 1: stimulatory effects on blood monocytes and monocyte-derived cells of the brain. Cancer Manag Res 2012;4:309-23.

25. Bilan PJ, Samokhvalov V, Koshkina A, et al. Direct and macrophage-mediated actions of fatty acids causing insulin resistance in muscle cells. Arch Physiol Biochem 2009;115:176-90.

26. $\mathrm{Yu}$ J, Shi L, Wang $\mathrm{H}$, et al. Conditioned medium from hypoxia-treated adipocytes renders muscle cells insulin resistant. Eur J Cell Biol 2011;90:1000-15.

27. Varma V, Yao-Borengasser A, Rasouli N, et al. Muscle inflammatory response and insulin resistance: synergistic interaction between macrophages and fatty acids leads to impaired insulin action. $A m \mathrm{~J}$ Physiol Endocrinol Metab 2009;296:E1300-10.

28. Weisberg SP, McCann D, Desai M, et al. Obesity is associated with macrophage accumulation in adipose tissue. J Clin Invest 2003;112:1796-808.

29. Kudo H, Yata Y, Takahara T, et al. Telmisartan attenuates progression of steatohepatitis in mice: role of hepatic macrophage infiltration and effects on adipose tissue. Liver Int 2009;29:988-96.

30. Odegaard JI, Chawla A. Mechanisms of macrophage activation in obesity-induced insulin resistance. Nat Clin Pract Endocrinol Metab 2008;4:619-26.

31. Samaan MC. The macrophage at the intersection of immunity and metabolism in obesity. Diabetol Metab Synd 2011;3:29.

32. Kraegen EW, Cooney GJ. Free fatty acids and skeletal muscle insulin resistance. Curr Opin Lipidol 2008;19:235-41.

33. Samaan MC. The macrophage at the intersection of immunity and metabolism in obesity. Diabetol Metab Syndr 2011;3:29.

34. Ingram $\mathrm{KH}$, Lara-Castro $\mathrm{C}$, Gower BA, et al. Intramyocellular lipid and insulin resistance: differential relationships in European and African Americans. Obesity 2011;19:1469-75.

35. Hulver MW, Berggren JR, Cortright RN, et al. Skeletal muscle lipid metabolism with obesity. Am J Physiol Endocrinol Metab 2003;284: E741-7.

36. Lipshultz SE, Landy DC, Lopez-Mitnik G, et al. Cardiovascular status of childhood cancer survivors exposed and unexposed to cardiotoxic therapy. J Clin Oncol 2012;30:1050-7.

37. Skinner AC, Steiner MJ, Henderson FW, et al. Multiple markers of inflammation and weight status: cross-sectional analyses throughout childhood. Pediatrics 2010;125:e801-9.
38. McVean JJ, Carrel AL, Eickhoff JC, et al. Fitness level and body composition are associated with inflammation in non-obese children. $J$ Pediatr Endocrinol Metab 2009;22:153-9.

39. Samaan MC, Obeid J, Nguyen T, et al. Chemokine (C-C motif) Ligand 2 is a potential biomarker of inflammation \& physical fitness in obese children: a cross-sectional study. BMC Pediatr 2013;13:47.

40. Amati L, Marzulli G, Martulli M, et al. Effects of a hypocaloric diet on obesity biomarkers: prevention of low-grade inflammation since childhood. Curr Pharm Des 2010;16:893-7.

41. Garanty-Bogacka B, Syrenicz M, Syrenicz A, et al. Serum markers of inflammation and endothelial activation in children with obesity-related hypertension. Neuro Endocrinol Lett 2005;26:242-6.

42. Mangge $\mathrm{H}$, Schauenstein $\mathrm{K}$, Stroedter $\mathrm{L}$, et al. Low grade inflammation in juvenile obesity and type 1 diabetes associated with early signs of atherosclerosis. Exp Clin Endocrinol Diabetes 2004;112:378-82.

43. Dedoussis GV, Kapiri A, Samara A, et al. Visfatin: the link between inflammation and childhood obesity. Diabetes Care 2009;32:e71.

44. Castro C, Tracy RP, Deckelbaum RJ, et al. Adiposity is associated with endothelial activation in healthy $2-3$ year-old children. J Pediatr Endocrinol Metab 2009;22:905-14.

45. Calcaterra V, De Amici M, Klersy C, et al Adiponectin, IL-10 and metabolic syndrome in obese children and adolescents. Acta Biomed 2009;80:117-23.

46. Samaan MC, Thabane L, Burrow S, et al. Canadian Study of Determinants of Endometabolic Health in ChIIDrEn (CanDECIDE study): a cohort study protocol examining the mechanisms of obesity in survivors of childhood brain tumours. BMJ Open 2013;3:pii: e002869.

47. Rockett HR, Breitenbach M, Frazier AL, et al. Validation of a youth/ adolescent food frequency questionnaire. Prev Med 1997;26:808-16.

48. https://regepi.bwh.harvard.edu/health/KIDS/files/03.\%202012\% 20 Youth\%20Adolescent\%20Food\%20Frequency\%20Questionnaire. pdf (accessed 9 May 2013).

49. Hay J, Cairney J. Development of the Habitual Activity Estimation Scale for clinical research: a systematic approach. Pediatr Exerc Sci 2006;18:193-202.

50. Chervin RD, Hedger K, Dillon JE, et al. Pediatric sleep questionnaire (PSQ): validity and reliability of scales for sleep-disordered breathing, snoring, sleepiness, and behavioral problems. Sleep Med 2000;1:21-32.

51. Faulstich ME, Carey MP, Ruggiero L, et al. Assessment of depression in childhood and adolescence: an evaluation of the Center for Epidemiological Studies Depression Scale for Children (CES-DC). Am J Psychiatry 1986;143:1024-7.

52. Brownson RC, Chang JJ, Eyler AA, et al. Measuring the environment for friendliness toward physical activity: a comparison of the reliability of 3 questionnaires. Am J Public Health 2004:94:473-83.

53. Coughlin SR, Mawdsley L, Mugarza JA, et al. Obstructive sleep apnoea is independently associated with an increased prevalence of metabolic syndrome. Eur Heart J 2004;25:735-41.

54. Yao M, Ludwa I, Corbett L, et al Bone speed of sound and physical activity levels of overweight and normal-weight girls and adolescents. Pediatr Exerc Sci 2011;23:25-35.

55. Harris PA, Taylor R, Thielke R, et al. Research electronic data capture (REDCap) - a metadata-driven methodology and workflow process for providing translational research informatics support. J Biomed Inform 2009;42:377-81. 